\title{
Online Adaptive Optimal Control for Continuous-Time Markov Jump Linear Systems Using A Novel Policy Iteration Algorithm*
}

\author{
Shuping $\mathrm{He}^{a \dagger}{ }^{\dagger}$ Jun Song ${ }^{a}$, Zhengtao Ding ${ }^{b}, \mathrm{Fei} \mathrm{Liu}^{c}$

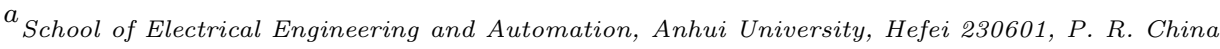 \\ ${ }^{b}$ Control Systems Center, School of Electrical and Electronic Engineering, University of Manchester, \\ Sackville Street Building, Manchester M13 9PL, UK \\ ${ }^{c}$ Key Laboratory of Advanced Process Control for Light Industry (Ministry of Education), Institute of Automation, \\ Jiangnan University, Wuxi, 214122, P.R. China
}

\begin{abstract}
This paper studies the online adaptive optimal control problems for a class of continuous-time Markov Jump Linear Systems (MJLSs) based on a novel policy iteration algorithm. By utilizing a new decoupling technique named Subsystems Transformation, we re-construct the MJLSs and a set of new coupled systems composed of $N$ subsystems are obtained. The online policy iteration algorithm was used to solve the coupled algebraic matrix Riccati equations with partial knowledge regarding to the system dynamics, and the relevant optimal controllers equivalent to the investigated MJLSs are designed. Moreover, the convergence of the novel policy iteration algorithm is also established. Finally, a simulation example is given to illustrate the effectiveness and applicability of the proposed approach.
\end{abstract}

Keywords: Markov Jump Linear Systems (MJLSs); Adaptive Optimal Control; Subsystems Transformation; Policy Iteration (PI); Online.

\section{Introduction}

Over the past few decades, Reinforcement Learning (RL) and Approximate/Adaptive Dynamic Programming (ADP) have been widely applied for solving the optimal control problems for linear/nonlinear systems with unknown or uncertain parametric models [41]. RL means finding an admissible control policy, i.e., learning the parameters of a controller mapping between the system states and the control signal, such that to maximize a numerical reward signal [38]. It is noted that the integral over time of the reward signal can be viewed as the value/cost function to be maximized/minimized in an optimal control framework. From the viewpoint of control engineering, RL algorithms can be viewed as a class of adaptive controllers which solve the optimal control

${ }^{*}$ This work was supported in part by the National Natural Science Foundation of P. R. China (Grant No. 61203051), the Key Program of Natural Science Foundation of Education Department of Anhui Province (Grant No. KJ2012A014) and the Joint Specialized Research Fund for the Doctoral Program of Higher Education (Grant No. 20123401120010).

${ }^{\dagger}$ Corresponding author. Tel: +86 0551 3861413, fax: +86 05513861413. Email addresses: shuping.he@ahu.edu.cn(S. He), jun_song@mail.ecust.edu.cn (J. Song), zhengtao.ding@manchester.ac.uk (Z. Ding), fliu@jiangnan.edu.cn (F. Liu). 
problem based on reward information which gives information on the performance of a given controller [37][42].

One class of RL algorithms is Policy Iteration (PI) which was first introduced in the computational intelligence community in the framework of stochastic decision theory [43]. PI algorithms iterate on two-steps named policy evaluation, in which the cost (i.e. predefined value function) associated with an admissible control policy is evaluated, and policy improvement, when the policy is updated such that will have a lower associated cost. The two-steps are forced to repeat until the policy improvement step no longer changes the present policy, thus convergence to the optimal controller is achieved [39][40].

Recently, a new RL method, namely Integral Reinforcement Learning (IRL), was provided to learn solution online to optimal control problem without requiring knowledge of the system drift dynamics. In [6], the authors employed this approach to solve the optimal control problem for continuous-time linear time-invariant systems using partial knowledge regarding to the system dynamics [6]. Thereafter, the approach was then extended to similar systems with completely unknown system dynamics in [7]. For an overview of contributions for linear systems, see [21][5]. The extension to the nonlinear field was given by [29][28][15][27][26].

In the work of [29], the authors studied an online algorithm based on PI for learning the continuous-time optimal control solution with infinite horizon cost with known dynamics. For the infinite-horizon optimal control problem for continuous-time uncertain nonlinear systems, Bhasin et al. have proposed an online adaptive reinforcement learning-based solution in [27]. The most important advantage of using ADP technique to get the solution of the optimal controller associated with linear/nonlinear systems is that this approach can be implemented online effective with partial system dynamics.

On another research front line, MJLSs have been widely studied by many researchers since the pioneering work of Krasovskii and Lidskii on quadratic control [12]; see, e.g., [13][8][23][22][25]. As an active research topic of control problem for MJLSs ,the optimal controller design problems have also regained increasing interest, as evidenced by several publications for related results [24][31]. In fact, the problem to solve the jump linear quadratic (JLQ) optimal controller (i.e. $H_{2}$ control [32]) for MJLSs is equivalent to finding the unique positive definition sequence solutions of a set of coupled algebraic Riccati equations [8][10]. In [10], Borno constructed a sequence of Lyapunov algebraic equations whose solutions converge to the solutions of the coupled algebraic Riccati equations of the optimal control problem for MJLSs. The algorithm was named as parallel algorithm, and similar results have been presented in [1][2][4]. Thereafter, Zhou et al. developed the gradient-based algorithm to solve the coupled discrete-time Markovian jump Lyapunov matrix equations [11]; Li et al. proposed a positive operator based iteration algorithms for solving Lyapunov equations for Itô stochastic systems with Markovian jumps [19]. It is easy to find that all of the aforementioned approaches are off-line algorithms for solving the coupled algebraic Riccati equations or coupled algebraic Lyapunov equations. Moreover, they also require the exactly information of the system dynamics.

However, to the best of our knowledge, the relevant research focusing on the adaptive control problem for MJLSs by means of ADP technique has not fully been researched in the literature, saying nothing of the online optimal controller design. In fact, different with regular linear/nonlinear systems, MJLSs have two components which are the jumping modes and continuous states, modeled by finite state Markov chains and differential equations. The variables not only associate with continuous-time domain but also depend on the probability space, thus the ADP algorithm proposed in regular linear/nonlinear systems can not be used for MJLSs by a simple extension.

In this paper, our aim is to develop a new iterative algorithm based on ADP for solving the JLQ optimal control problem for continuous-time MJLSs. The novel algorithm solves the optimal control problem for continuous-time 
MJLSs only use partial knowledge regarding to the system dynamics. Inspired by the parallel algorithm [10][35][4] and a new decoupling method named Subsystems Transformation, we re-construct a set of new continuous-time Markov jump linear subsystems and separate the coupled relation from the coupled algebraic matrix Riccati equations based on the jumping parameters. We also show that the optimal controllers of the constructed subsystems with the explicit parameters equal to the investigated continuous-time MJLSs. Subsequently, based on the framework of two-step policy iteration, a novel iterative algorithm which can be implementation online are studied and the convergence of the algorithm is also proved. In order to illustrate the proposed results, we provide a fourth-order jump linear models, presented by [2][4]. The obtained simulation results demonstrate the feasibility and validity of the new approach.

This paper is organized as follows: The problem formulation and preliminaries are given in Section 2. A new decoupling technique named Subsystems Transformation is proposed in Subsection 3.1. The novel iterative algorithm is presented in Subsection 3.2 and the proof of convergence is shown in Subsection 3.3. In Section 4 , a simulation example is given to demonstrate the capabilities of the proposed novel policy iteration scheme. Conclusions are presented in Section 5.

Notations. Throughout this paper, we use the following notations: $\mathbf{R}^{n}$ and $\mathbf{R}^{n \times m}$ stand for an $n$-dimensional Euclidean space and the set of all $n \times m$ real matrices, respectively; $\lambda_{\max }(P)$ and $\lambda_{\min }(P)$ denote the maximal and minimal eigenvalues of a positive-define matrix $P ; \mathrm{E}\{*\}$ denotes the statistical expectation of the stochastic process or vector; $P<0$ or $P>0$ means matrix $P$ is negative or positive-definite; $I$ and 0 are respectively the unit and the zero matrices with appropriate dimensions.

\section{Problem Formulation and Preliminaries}

\subsection{Continuous-Time MJLSs and JLQ Optimal Problem}

Consider the following continuous-time linear systems with Markovian jumps in the probability space $(\Omega, \mathcal{F}, \mathcal{P})$ $[12][9]$ :

$$
\dot{x}(t)=A(r(t)) x(t)+B(r(t)) u(t)
$$

where $\Omega$ is the sample space, $\mathcal{F}$ is the algebra of events, and $\mathcal{P}$ is the probability measure defined on $\mathcal{F} ; x(t) \in \mathbf{R}^{n}$ is the state vector, and $u(t) \in \mathbf{R}^{m}$ is the controlled input; $A(r(t))$ and $B(r(t))$ are known mode-dependent matrices with appropriate dimensions; $\{r(t), t \geq 0\}$ is a continuous-time discrete-state Markovian process with left continuous trajectories [13] and taking values from a finite set $\mathbf{S}=\{1,2, \cdots, N\}$ with transition probabilities given by

$$
P_{r}\{r(t+\Delta t)=j \mid r(t)=i\}=\left\{\begin{array}{cc}
\pi_{i j} \Delta t+o(\Delta t) & j \neq i, \\
1+\pi_{i i} \Delta t+o(\Delta t) & j=i,
\end{array}\right.
$$

where $\Delta t>0, \lim _{\Delta t \rightarrow 0} \frac{o(\Delta t)}{\Delta t}=0$, and $\pi_{i j} \geq 0(j \neq i)$ is the transition rate from mode $i$ at time $t$ to mode $j$ at time $t+\Delta t$ and

$$
\pi_{i i}=-\sum_{j \in \mathbf{S}, j \neq i} \pi_{i j}
$$

For convenience, we denote $A(r(t)), B(r(t)), Q(r(t)), R(r(t))$ as $A_{i}, B_{i}, Q_{i}, R_{i}$ respectively with $r(t)=i, i \in \mathbf{S}$.

Subject to system (1)-(2) which is assumed to be stochastically stabilizable [8], our objective is to find a set of mode-dependent control policy $\psi(t, x(t), i) \in \Psi$, i.e.

$$
u_{i}(t) \triangleq \psi(t, x(t), i), \psi:\left[t_{0}, \infty\right) \times \mathbf{R}^{n} \times \mathbf{S} \rightarrow \mathbf{R}^{m},
$$


which minimizes the following performance criterion

$$
V\left(t, x(t), r(t)=i, u_{i}\right)=\mathrm{E}\left\{\int_{t_{0}}^{\infty}\left[x^{\mathrm{T}}(t) Q_{i} x(t)+u^{\mathrm{T}}(t) R_{i} u(t)\right] \mathrm{d} t \mid x\left(t_{0}\right), r\left(t_{0}\right), \forall t_{0} \geq 0\right\}
$$

where the mode-dependent weighting matrices $Q_{i}$ and $R_{i}$ are real valued with $Q_{i}^{\mathrm{T}}=Q_{i} \geq 0, R_{i}^{\mathrm{T}}=R_{i}>0$ $(\forall i \in \mathbf{S})$. Note that the mode-dependent control policy $u_{i}(t)$ must not only stochastic stabilize the MJLSs on $\mathbf{R}^{m}$ but also guarantee that (5) is finite for all $i \in \mathbf{S}$. That is to say, mode-dependent control policy $u_{i}(t)$ must be admissible for all $i \in \mathbf{S}$. To this end, the definition of admissible control policy for the regular linear/nonlinear [33][18] is generalized to MJLSs as follows.

Definition 1. (Mode-Dependent Admissible Control Policy) For all $i \in \mathbf{S}$, the mode-dependent control policy $u_{i}(t)=\psi(t, x(t), i)$ with $\psi\left(t_{0}, x\left(t_{0}\right), r\left(t_{0}\right)\right) \equiv 0$ is said to be mode-dependent admissible with respect to (5) on $\Psi$, if $\psi(t, x(t), i)$ is continuous on a compact set $\Psi \subseteq \mathbf{R}^{m}$, stochastic stabilizes the MJLSs (1)-(2), and yields a finite mode-dependent value function $\left.V_{i}\left(x(t), u_{i}(t)\right)=\mathrm{E}\left\{V\left(t, x(t), r(t)=i, u_{i}(t)\right)\right\} \mid t_{0}, x\left(t_{0}\right), r\left(t_{0}\right)\right\}\left.\right|_{\psi(t, x(t), i)}$.

Then, the JLQ optimal control problem for continuous-time MJLSs (1)-(2) is equivalent to find the following a set of mode-dependent admissible control policy:

$$
u_{i}(t)=u_{i}^{*}(x(t)) \triangleq \arg \min _{u_{i} \in \Psi} V_{i}\left(x(t), u_{i}(t)\right), \forall i \in \mathbf{S}
$$

\subsection{Preliminaries}

Lemma 1. (Ji \& Chizeck [8]; Costa, do Val $\& 3$ Geromel [32]) For JLQ optimal problem (1)-(6), the modedependent optimal steady state control policy is

$$
u_{i}(t)=-K_{i} x(t)=-R_{i}^{-1} B_{i}^{\mathrm{T}} P_{i} x(t),
$$

where $P_{i}(i \in \mathbf{S})$ is the set of unique, positive solutions of the following coupled algebraic matrix Riccati equations

$$
A_{i}^{\mathrm{T}} P_{i}+P_{i} A_{i}-P_{i} B_{i} R_{i}^{-1} B_{i}^{\mathrm{T}} P_{i}+\sum_{j=1}^{N} \pi_{i j} P_{j}+Q_{i}=0 .
$$

And the controlled system is mean square stable in the sense that

$$
\mathrm{E}\left\{\|x(t)\|^{2} \mid x_{0}, r_{0}\right\} \rightarrow 0 \quad(\text { as } t \rightarrow \infty) .
$$

It is seen from Lemma 1 that the optimal control problem for MJLSs is reduced to finding a set of stochastically stabilizing solutions $P_{i}$ of the coupled algebraic matrix Riccati equations (8). Based on the Newton-RaphsonKantorovich method [3] or Kleinman's method [14], the solution of (7) can be obtained [4].

In [1], Borno used parallel algorithm to solve continuous-time MJLSs of coupled Lyapunov equations. Thereafter, in [10], Gajic and Borno studied a new computation iteration method to solve the optimal control problem for continuous-time MJLSs based on parallel algorithm (the algorithm is introduced in Lemma 2). In fact, the biggest advantage of utilizing the parallel algorithm to solve the above problems is that, both the coupled algebraic Riccati equations and coupled algebraic Lyapunov equations are reduced to a class of decoupled algebraic Lyapunov equations. 
Assumption 1. (Gajic \& Borno [2]) The triples $\left(A_{i}, B_{i}, \sqrt{Q_{i}}\right), i \in \mathbf{S}$ are stabilizable-detectable, and

$$
\max _{i \in \mathbf{S}}\left\{i n f_{\Gamma_{i}}\left|\lambda_{\max }\left[\int_{0}^{\infty} e^{\Upsilon_{i}^{\mathrm{T}}} t \times e^{\Upsilon_{i}} t \mathrm{~d} t\right]\right|\right\}<1
$$

where $\Upsilon_{i}=A_{i}+B_{i} \Gamma_{i}+\frac{\pi_{i i}}{2} I, \Gamma_{i}$ are arbitrary real matrices.

Condition (10) is a consequence of the fixed point iterations [34] used to establish the existence and uniqueness of the solutions of the proposed parallel iteration algorithm in the following Lemma 2. Actually, Assumption 1 guarantees stabilizability of the matrices $\left(A_{i}+\frac{\pi_{i i}}{2} I\right)$ by the optimal closed-loop feedback gain. In [35], Gajic and Losada have pointed out that the Assumption 1 is equivalent to the following assumption.

Assumption 2. (Gajic \& Losada [35]; Borno [1]) The continuous-time MJLSs (1)-(2) are stochastically stabilizable and the pairs $\left(A_{i}, \sqrt{Q_{i}}\right), i \in \mathbf{S}$, are deterministically detectable.

Lemma 2. (Gajic \& Borno [2]) Let Assumption 1 (or Assumption 2) hold. Then, the solution of the following constructed sequence of Lyapunov algebraic equations converges to the solutions of the coupled algebraic Riccati equations (8):

$$
\left(\mathbf{A}_{i}-B_{i} R_{i}^{-1} B_{i}^{\mathrm{T}} P_{i}(k)\right)^{\mathrm{T}} P_{i}(k+1)+P_{i}(k+1)\left(\mathbf{A}_{i}-B_{i} R_{i}^{-1} B_{i}^{\mathrm{T}} P_{i}(k)\right)=-P_{i}(k) B_{i} R_{i}^{-1} B_{i}^{\mathrm{T}} P_{i}(k)-Q_{i}(k)
$$

with stabilizing $P_{i}(0) \geq 0, i \in \mathbf{S}$ and where

$$
\left\{\begin{array}{l}
\mathbf{A}_{i}=A_{i}+\frac{\pi_{i i}}{2} I \\
Q_{i}(k)=Q_{i}+\sum_{j=1, j \neq i}^{N} \pi_{i j} P_{j}(k) \geq 0
\end{array}\right.
$$

Remark 1. It is easy to see that at every iterative step of the algorithm (11), $N$ represents the continuous-time Lyapunov equations with the form

$$
A X+X A^{\mathrm{T}}=-Q, Q>0,
$$

to be solved. At present, many numerically algorithms for solving the aforementioned equations have been imbedded in the MATLAB functions, such as, lyap and are [16][17].

As having been pointed out in [18][6][7][5], all of the above mentioned computational iteration algorithms are offline methods for obtaining the solution of the coupled algebraic matrix Riccati equations (8). In these algorithms, the exact knowledge of system coefficient matrices $A_{i}(\forall i \in \mathbf{S})$ are required.

However, in many practical applications, the real system dynamics are always unknown, that is, the system is completely model-free or partially model-free. To overcome this limitation, some adaptive control techniques have be proposed by many researchers. For a continuous-time linear time-invariant dynamical system, a new policy iteration algorithm was presented for online finding the solutions of the optimal control problem without using the system matrix by Vrabie et al. [6]. This new policy iteration algorithm is in the following.

Lemma 3. (Vrabie et al. [6]) For a linear time-invariant system described by: $\dot{x}(t)=A x(t)+B u(t)$, the solution of the infinite horizon quadratic optimal control problem is given by $u(t)=-K x(t)$ with

$$
K=R^{-1} B^{\mathrm{T}} P,
$$

where the matrix $P$ is the unique real symmetric positive definite solution of the Lyapunov matrix equation

$$
[A-B K]^{\mathrm{T}} P+P[A-B K]=-\left[K^{\mathrm{T}} R K+Q\right] .
$$


Then, denoting $x(t)$ with $x_{t}$, the following two-step policy iteration algorithm can efficiently solve the above Lyapunov matrix equation online with an initial stabilizing controller gain $K(0)$ :

Policy Evaluation:

$$
x_{t}^{\mathrm{T}} P(k) x_{t}=\int_{t}^{t+T} x_{\tau}^{\mathrm{T}}\left[Q+K(k)^{\mathrm{T}} R K(k)\right] x_{\tau} \mathrm{d} \tau+x_{t+T}^{\mathrm{T}} P(k) x_{t+T},
$$

Policy Improvement:

$$
K(k+1)=R^{-1} B^{\mathrm{T}} P(k) .
$$

\section{Main Results}

In this paper, we propose a novel policy iteration algorithm that will online solve the optimal control problem for a class of continuous-time MJLSs only using partial knowledge regarding to the system dynamics. The new algorithm is based on parallel algorithm [1][10] and subsystems transformation [30]. At each iteration step of the new algorithm, $N$ reconstructed parallel subsystems all required to execute a two-step iteration namely the Critic update and the Actor update. Proof of convergence of the algorithm is also provided in this section.

\subsection{Subsystems Transformation}

In fact, the exact information of the MJLSs coefficient matrices $A_{i}, B_{i}$ and the transition matrix elements $\pi_{i j}$ are all required in solving the coupled algebraic matrix Riccati equations (8). When we directly observe the states online, the information regarding $\pi_{i j}$ are not contained in the observed states. Therefore, the existing online policy iteration schemes cannot be applied for solving the JLQ optimal control problems of the MJLSs. To overcome this shortcoming, we give the following Theorem 1. By reconstructing the continuous-time MJLSs, we separate the information of $\pi_{i j}$ from the MJLSs. And the information regarding the system coefficient matrices $A_{i}$ are embedded in the reconstructed subsystems states which can be observed online.

Theorem 1. (Subsystems Transformation) Let $P_{i}(k)$ be the solutions of the following ith continuous-time linear reconstructed subsystems

$$
\dot{x}_{i, t}=\mathbf{A}_{i} x_{i, t}+B_{i} u_{i, t}(i=1,2, \cdots, N),
$$

at kth Kleinman iteration step with the parameter $Q_{i}$ that satisfy:

$$
Q_{i}(k)=Q_{i}+\sum_{j=1, j \neq i}^{N} \pi_{i j} P_{j}(k-1) .
$$

Then, the following property hold:

$$
\lim _{k \rightarrow \infty} P_{i}(k)=P_{i}^{*}, \forall i \in \mathbf{S}
$$

where the $P_{i}^{*}$ are the solutions of (8).

Proof. For the constructed continuous-time linear system (18), the solution of the Kleinman iteration with (19) equals the solution of the following equation at each iteration step [14][7]:

$$
\left[\mathbf{A}_{i}-B_{i} K_{i}(k)\right]^{\mathrm{T}} P_{i}(k)+P_{i}(k)\left[\mathbf{A}_{i}-B_{i} K_{i}(k)\right]+Q_{i}(k)+K_{i}^{\mathrm{T}}(k) R_{i} K_{i}(k)=0,
$$

where $K_{i}(k)=R_{i}^{-1} B_{i}^{\mathrm{T}} P_{i}(k-1)$. 
It is easy to see that (21) is equivalent to (11). Hence, according to Lemma 2, we can obtain (20). This completes the proof.

Remark 2. By reconstructing the continuous-time $M J L S s$, the exact information regarding $A_{i}(\forall i \in \mathbf{S})$ are contained in system matrices $\mathbf{A}_{i}$ of $N$ parallel continuous-time linear subsystems in form of (18).

\subsection{A novel policy iteration algorithm}

By utilizing the parallel algorithm and subsystems transformation, the continuous-time MJLSs (1)-(3) are decomposed into $N$ interrelated continuous-time linear time-invariant subsystems in form of (18), and each optimal controller can be obtained by the iteration algorithm (11)-(12). In Remark 1, we have pointed out that, at every iteration step, the iteration solution can be obtained from a standard Lyapunov equation in form of (13). Combining Lemma 3, we devise the following new policy iteration algorithm:

\section{Policy Evaluation:}

$$
\left\{\begin{array}{c}
x_{1, t}^{\mathrm{T}} P_{1}(k) x_{1, t} \\
=\int_{t}^{t+T} x_{1, \tau}^{\mathrm{T}}\left[Q_{1}(k)+K_{1}^{\mathrm{T}}(k) R_{1} K_{1}(k)\right] x_{1, \tau} \mathrm{d} \tau \\
+x_{1, t+T}^{\mathrm{T}} P_{1}(k) x_{1, t+T}, \\
\vdots \\
x_{i, t}^{\mathrm{T}} P_{i}(k) x_{i, t} \\
=\int_{t}^{t+T} x_{i, \tau}^{\mathrm{T}}\left[Q_{i}(k)+K_{i}^{\mathrm{T}}(k) R_{i} K_{i}(k)\right] x_{i, \tau} \mathrm{d} \tau \\
+x_{i, t+T}^{\mathrm{T}} P_{i}(k) x_{i, t+T}, \\
\vdots \\
x_{N, t}^{\mathrm{T}} P_{N}(k) x_{N, t} \\
=\int_{t}^{t+T} x_{N, \tau}^{\mathrm{T}}\left[Q_{N}(k)+K_{N}^{\mathrm{T}}(k) R_{N} K_{N}(k)\right] x_{N, \tau} \mathrm{d} \tau \\
+x_{N, t+T}^{\mathrm{T}} P_{N}(k) x_{N, t+T},
\end{array}\right.
$$

\section{Policy Improvement:}

$$
\begin{aligned}
& Q_{i}(k+1)=Q_{i}+\sum_{j=1, j \neq i}^{N} \pi_{i j} P_{j}(k), \\
& K_{i}(k+1)=R_{i}^{-1} B_{i}^{\mathrm{T}} P_{i}(k) .
\end{aligned}
$$

with stabilizing sequences $\left\{P_{i}(0)\right\} \geq 0(\forall i \in \mathbf{S})$.

Note that the system matrices $A_{i}$ and the transfer matrix elements $\pi_{i i}$ are not included in implementing this algorithm. But in the policy improvement equations (23)-(24), the exact knowledge of the system matrices $B_{i}$ and the transfer matrix elements $\pi_{i j}$ are still required for the iterations. Therefore, this proposed policy iteration algorithm is a partially mode-free adaptive dynamic programming algorithm.

Remark 3. The information regarding to the system matrices $A_{i}$ are embedded in the subsystems state trajectories $x_{i, t}$ which can be observed online. According to (3), it is easy to see that the information of $\pi_{i i}$ is also included in $\pi_{i j}$ by the proposed policy iteration scheme. 
Remark 4. In the policy update equation (23), it contains all the of coupling relation information of the coupled algebraic matrix Riccati equations (8). Thus, the exact knowledge of state transfer matrix $\left\{\pi_{i j}\right\}$ is required in either off-line algorithms (such as, [1][2][19]) or online algorithm (22)-(23).

\subsection{Proof of convergence and online implementation}

Theorem 2. Under the assumptions of the system matrices $A_{i}$ are stablilizable, the condition equation (10) is hold for arbitrary real matrices $\Gamma_{i}$, and the new policy iteration (22)-(24) converges to the optimal controller given by (7), wherein the matrices $P_{i}$ is the unique real symmetric positive definite solution of the coupled algebraic matrix Riccati equations (8).

Proof. For any $i$ th subsystem described as

$$
\dot{x}_{i, t}=\mathbf{A}_{i} x_{i, t}+B_{i} u_{i, t}
$$

Obviously, the system is a continuous-time linear time-variant dynamical one. Along with the state trajectories of the dynamical system (25), we operate the new adaptive iteration algorithm (22)-(24). Letting $k=0$ and giving initial stabilizing sequences $\left\{P_{i}(0)\right\}(i=1,2, \cdots, N)$, we start the policy iteration scheme by using policy improvement (23)-(24), then one can obtain the following solutions:

$$
\begin{aligned}
& Q_{i}(1)=Q_{i}+\sum_{j=1, j \neq i}^{N} \pi_{i j} P_{j}(0), \\
& K_{i}(1)=R_{i}^{-1} B_{i}^{\mathrm{T}} P_{i}(0)
\end{aligned}
$$

Substituting (26)-(27) into the policy evaluation (22), then it yields the solutions of $\left\{P_{i}(1)\right\}$.

According to Lemma 3 , the sequence $\left\{P_{i}(1)\right\}$ is also the solution of the underlying Lyapunov equation

$$
\left[\mathbf{A}_{i}-B_{i} K_{i}(1)\right]^{\mathrm{T}} P_{i}(1)+P_{i}(1)\left[\mathbf{A}_{i}-B_{i} K_{i}(1)\right]=-\left[K_{i}^{\mathrm{T}}(1) R_{i} K_{i}(1)+Q_{i}(1)\right]
$$

It is easy to see that (28) equals to (11) while $k=0$. Performing the same procedure, we can get the each iteration step solution $P_{i}(k)$ of the iteration algorithm (22)-(24), which is equivalent to the $k$ th iteration solution of the iteration algorithm (11).

From Lemma 2, we know that the solutions of computational algorithm (11) converge to the solutions of coupled algebraic matrix Riccati equations (8). This implies that the solutions of iteration algorithm (22)-(24) converge to the solutions of (8). This completes the proof.

Remark 5. In [4], the author has pointed out that the iteration algorithm (10) equals to the standard Newton's algorithm [14][3][20] with the form:

$$
P_{i}(k+1)=P_{i}(k)-\left(\mathcal{R}_{P_{i}(k)}^{\prime}\right)^{-1} \mathcal{R}\left(P_{i}(k)\right)
$$

where

$$
\mathcal{R}\left(P_{i}(k)\right)=A_{i}^{\mathrm{T}} P_{i}(k)+P_{i}(k) A_{i}-P_{i}(k) B_{i} R_{i}^{-1} B_{i}^{\mathrm{T}} P_{i}(k)+\sum_{j=1}^{N} \pi_{i j} P_{j}(k)+Q_{i},
$$

and the notation $\mathcal{R}_{P_{i}(k)}^{\prime}$ means the Fréchet derivative of $\mathcal{R}$ at point $P_{i}(k)$. Therefore, according to Theorem 2 , the proposed new iteration algorithm (22)-(24) is also equivalent to the Newton's method (29)-(30). 
Next, we derive the implement approach [6][5] for the new online iteration algorithm (22)-(24). It is necessary to point that the states of $N$ parallel subsystems are simultaneously online measured at each iteration step. To this end, we rewrite the term $x_{i, t}^{\mathrm{T}} P_{i}(k) x_{i, t}$ in $(22)$ as

$$
x_{i, t}^{\mathrm{T}} P_{i}(k) x_{i, t}=\left[\hat{p}_{i}(k)\right]^{\mathrm{T}} \hat{x}_{i}(t),
$$

where $\hat{x}_{i}(t)$ denotes the Kronecker product quadratic polynomial basis vector with the elements $\left\{x_{i, h}(t) x_{i, l}(t)\right\}$ $(h=1,2, \cdots, n ; l=h, h+1, \cdots, n)$, that is,

$$
\hat{x}_{i}=\left[x_{i, 1}^{2}, x_{i, 1} x_{i, 2}, \cdots, x_{i, 1} x_{i, n}, x_{i, 2}^{2}, x_{i, 2} x_{i, 3}, \cdots, x_{i, n}^{2}\right]
$$

and $\hat{p}_{i}(k)$ denotes the a column vector by stacking the elements of the diagonal and upper triangular part of the symmetric matrix $P_{i}$ into a vector where the off-diagonal elements are taken as $2 p_{i, h l}$, that is,

$$
\hat{p}_{i}=\left[p_{i, 11}, 2 p_{i, 12}, \cdots, 2 p_{i, 1 n}, p_{i, 22}, 2 p_{i, 23}, \cdots, p_{i, n n}\right]^{\mathrm{T}} .
$$

Then, (22) can be written as

$$
\left[\hat{p}_{i}(k)\right]^{\mathrm{T}}\left[\hat{x}_{i, t}-\hat{x}_{i, t+T}\right]=\int_{t}^{t+T} x_{i, \tau}^{\mathrm{T}}\left[Q_{i}(k)+K_{i}^{\mathrm{T}}(k) R_{i} K_{i}(k)\right] x_{i, \tau} \mathrm{d} \tau,
$$

In (34), for the $i$ th subsystem, at $k$ th iteration step, the regression vector $\hat{x}_{i, t}-\hat{x}_{i, t+T}$ and $\mathcal{R}\left(P_{i}(k)\right)$ can be obtained by online measuring the trajectories of the $i$ th subsystem. And $\hat{p}_{i}(k)$ is a vector of unknown parameters which can be obtained from (34). Notice that the symmetric matrix $P_{i}$ has $n(n+1) / 2$ unknown independent elements. Hence, we need at least $M(\geq n(n+1) / 2)$ independent equations to get $P_{i}(k)$. Therefore, for any $i$ th system, we should sample $M$ state vector in each time interval $T$. Then, the sequences $\left\{P_{i}(k)\right\}$ can be found in the following parallel least-square sense:

$$
\left\{\begin{array}{c}
\hat{p}_{1}(k)=\left[X_{1} X_{1}^{\mathrm{T}}\right]^{-1} X_{1} Y_{1}, \\
\vdots \\
\hat{p}_{i}(k)=\left[X_{i} X_{i}^{\mathrm{T}}\right]^{-1} X_{i} Y_{i}, \\
\vdots \\
\hat{p}_{N}(k)=\left[X_{N} X_{N}^{\mathrm{T}}\right]^{-1} X_{N} Y_{N},
\end{array}\right.
$$

where

$$
\begin{aligned}
& X_{i}=\left[\begin{array}{llll}
\hat{x}_{i}(t)-\hat{x}_{i}(t+\delta t) & \hat{x}_{i}(t+\delta t)-\hat{x}_{i}(t+2 \delta t) & \cdots & \hat{x}_{i}(t+(M-1) \delta t)-\hat{x}_{i}(t+M \delta t)
\end{array}\right], \\
& Y_{i}=\left[y_{i}^{(0)}(k) y_{i}^{(1)}(k) \cdots y_{i}^{(M-1)}(k)\right]^{\mathrm{T}},
\end{aligned}
$$

with $\delta t=T / M$ and $y_{i}^{(\mathrm{r})}(k)=\int_{t+r \delta t}^{t+(r+1) \delta t} x_{i, \tau}^{\mathrm{T}}\left[Q_{i}(k)+K_{i}^{\mathrm{T}}(k) R_{i} K_{i}(k)\right] x_{i, \tau} \mathrm{d} \tau(r=0,1, \cdots, M-1)$.

Remark 6. In order to have persistent excitation property of the data necessary to obtain the solution given by (35), one has to continue reading the states information from overall $N$ subsystems at each iterative step until the solution of the parallel least-squares sense is feasible. Here, we further point out that, before finding the ultimate optimal controller, the controller solutions obtained at each step by (24) are all suboptimal statefeedback controllers for the concerned continuous-time MJLSs. Namely, these obtained suboptimal state-feedback controllers be able to control the states of the MJLSs reach to zeros in finite time interval but the performance criterions computed by using the information of the closed-loop systems that are controlled by these suboptimal controllers are not the minimal one. 
Figure 1: Subsystems Transformation and Policy Iteration.

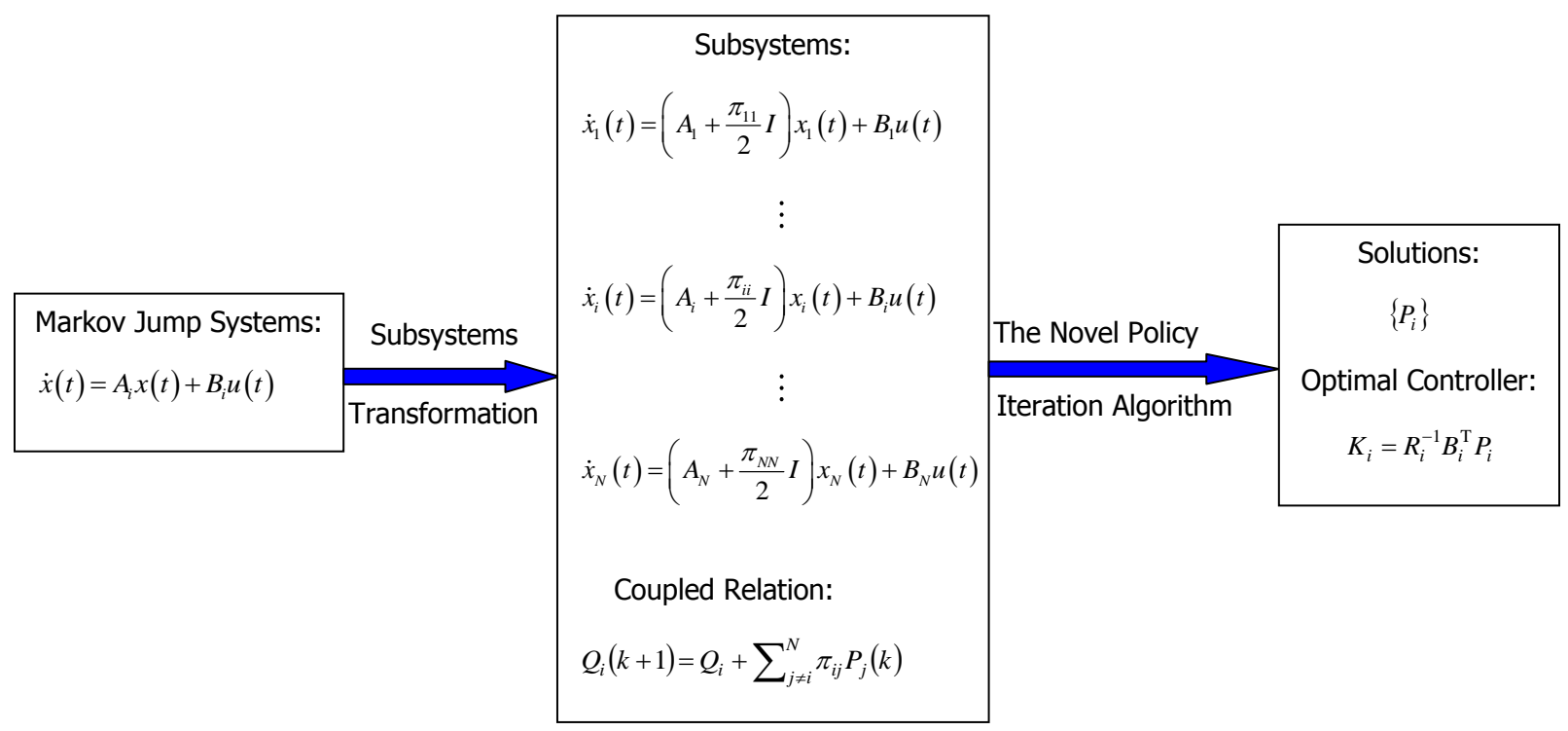

Remark 7. It is necessary to point out that there is no significant relationship between the each iteration time interval $T$ in (22) and the jumping time constant $\Delta t$ of the continuous-time MJLSs (1)-(3). We need to point out that the the continuous-time MJLSs (1)-(3) can be regarded as a sequence of subsystems in form of (18). And the set of decoupled algebraic Lyapunov equations (11) converges to the coupled algebraic matrix Riccati equations (8). Using parallel algorithm and subsystems transformation, the coupled relation of (8) is expressed in the explicit formula (12) or (23). Subsystems Transformation and Policy Iteration are presented in Figure 1.

Remark 8. We define the following judging condition which need to be calculated at each iteration step of the implementation of the novel policy iteration algorithm (22)-(24):

$$
\max _{\forall i \in \mathbf{S}}\left(\left\|P_{i}(k+1)-P_{i}(k)\right\|\right) \leq \varepsilon,
$$

where $\varepsilon$ is the given error accuracy. The proposed novel policy iteration algorithm always execute until the stopping criterion (38) is satisfied. A flow chart of the novel policy iteration algorithm (22)-(24) is shown in Figure 2.

\section{Simulation Example}

In this section, we consider a fourth-order jump linear control problem [10][4] to illustrate the feasibility and validity of the proposed novel policy iteration algorithm. Using the proposed algorithm, the optimal control problem of MJLSs can be solved efficiently while the system matrices $A_{i}(i \in \mathbf{S})$ are unknown or not be utilized. 
Figure 2: The flowchart of policy iteration algorithm (22)-(24).

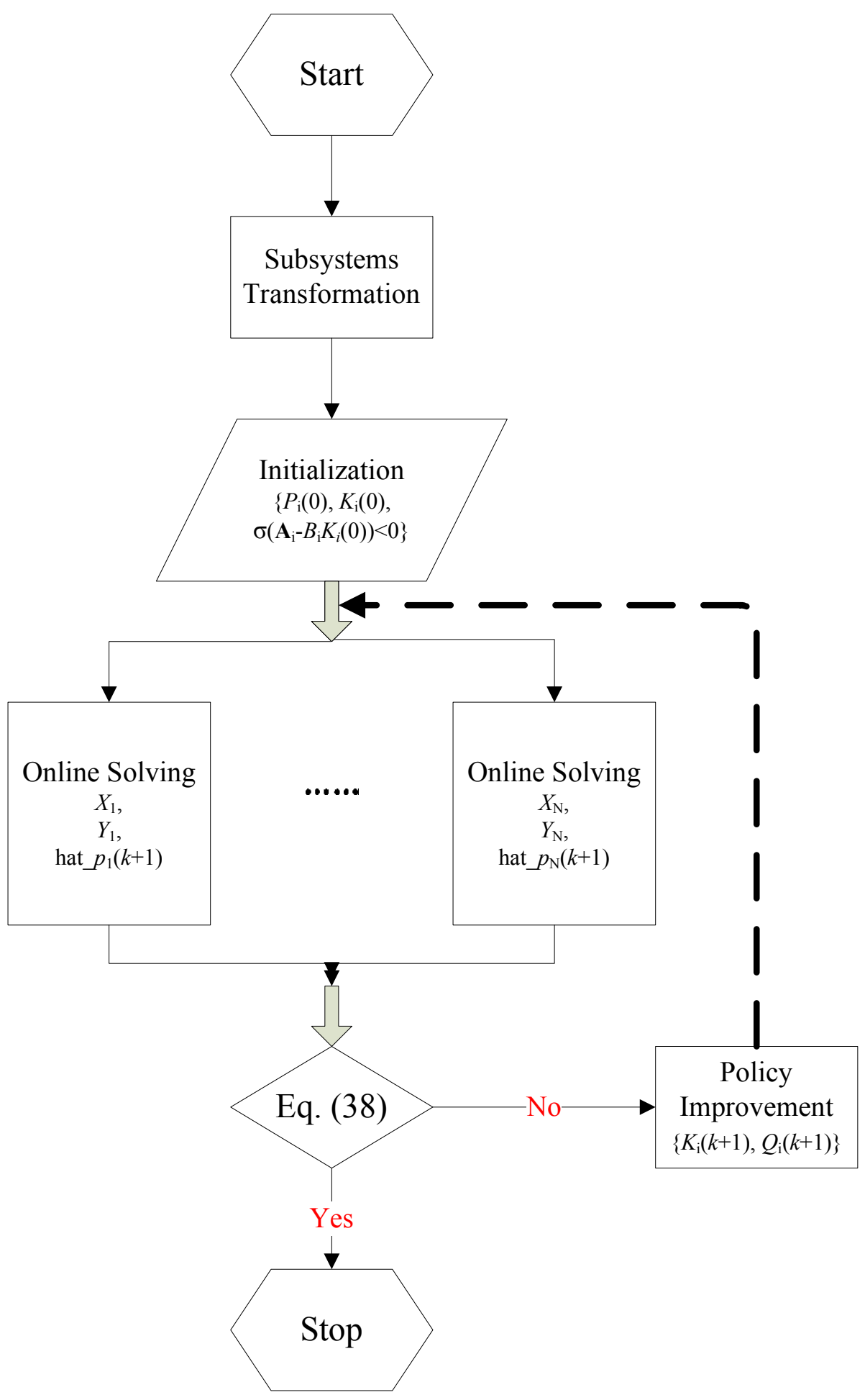


The matrix coefficients of the Markov jump linear system (1)-(3) are

$$
\begin{aligned}
& \Pi=\left[\begin{array}{cc}
-2 & 2 \\
1.5 & -1.5
\end{array}\right], \\
& A_{1}=\left[\begin{array}{cccc}
-2.1051 & -1.1648 & 0.9347 & 0.5194 \\
-0.0807 & -2.8949 & 0.3835 & 0.8310 \\
0.6914 & 10.5940 & -36.8199 & 3.8560 \\
1.0692 & 13.4230 & 22.1185 & -13.1801
\end{array}\right], B_{1}=\left[\begin{array}{l}
0.7564 \\
0.9910 \\
9.8255 \\
7.2266
\end{array}\right], \\
& A_{2}=\left[\begin{array}{cccc}
-2.6430 & -1.2497 & 0.5269 & 0.6539 \\
-0.7910 & -2.8570 & 0.0920 & 0.4160 \\
21.0357 & 22.8659 & -26.4655 & -1.7214 \\
27.3096 & 7.8736 & -3.8604 & -29.5345
\end{array}\right], B_{2}=\left[\begin{array}{l}
0.3653 \\
0.2470 \\
7.5336 \\
6.5152
\end{array}\right], \\
& Q_{1}=Q_{2}=\left[\begin{array}{cccc}
1 & 0 & 1 & 0 \\
0 & 0 & 0 & 0 \\
1 & 0 & 1 & 0 \\
0 & 0 & 0 & 0
\end{array}\right], R_{1}=R_{2}=1 .
\end{aligned}
$$

We set that the optimal controller is updated every $0.3 \mathrm{sec}$ and the two subsystems states information are conducted over each interval of 0.03sec. One thus have 10 sample data for every subsystem in each update interval. It should be noted that there are 10 independent elements in the symmetric matrices $P_{1}$ and $P_{2}$. Hence, at each update interval $0.3 \mathrm{sec}$ and for every reconstructed subsystem, the 10 sample data are enough for exciting to solving parallel least-square equations (35). In addition, we set the initial states of the two subsystems as $x_{1}(0)=x_{2}(0)=\left[\begin{array}{llll}0 & -0.1 & 0.1 & 0\end{array}\right]^{\mathrm{T}}$ and the value of $\varepsilon$ in $(38)$ is selected as $10^{-15}$.

The initial matrices $P_{1}(0)$ and $P_{2}(0)$ are chosen to be $I_{4 \times 4}$. At first, by solving policy improvement (23)(24), the corresponding matrices $Q_{i}(1)$ and $K_{i}(1)$ are obtained. Thereafter, executing the proposed novel policy iteration algorithm (22)-(24), the following $P_{1}$ and $P_{2}$ of the JLQ optimal control problem, without knowing the plant internal dynamics $A_{i}$, are found after 13 iterations:

$$
P_{1}(13)=\left[\begin{array}{llll}
0.2426 & 0.0675 & 0.0393 & 0.0180 \\
0.0675 & 0.0365 & 0.0086 & 0.0067 \\
0.0393 & 0.0086 & 0.0157 & 0.0025 \\
0.0180 & 0.0067 & 0.0025 & 0.0017
\end{array}\right], P_{2}(13)=\left[\begin{array}{llll}
0.5054 & 0.1312 & 0.0516 & 0.0097 \\
0.1312 & 0.0555 & 0.0135 & 0.0025 \\
0.0516 & 0.0135 & 0.0191 & 0.0004 \\
0.0097 & 0.0025 & 0.0004 & 0.0002
\end{array}\right] .
$$

Under the computational error $10^{-15}$, the following solutions of the coupled algebraic matrix Riccati equation for the fourth-order jump linear system have been solved by using Newton's iteration approach shown in [4] and the parallel iteration algorithm proposed in [2]:

$$
P_{1}^{*}=\left[\begin{array}{llll}
0.2408 & 0.0705 & 0.0393 & 0.0182 \\
0.0705 & 0.0308 & 0.0085 & 0.0064 \\
0.0393 & 0.0085 & 0.0157 & 0.0025 \\
0.0182 & 0.0064 & 0.0025 & 0.0016
\end{array}\right], P_{2}^{*}=\left[\begin{array}{llll}
0.5026 & 0.1343 & 0.0518 & 0.0097 \\
0.1343 & 0.0485 & 0.0138 & 0.0026 \\
0.0518 & 0.0138 & 0.0193 & 0.0002 \\
0.0097 & 0.0026 & 0.0002 & 0.0003
\end{array}\right] .
$$

The simulation results are shown in Figures 3-5. In Figures 3-4, the evolution of the complete parameters of the $P_{1}(k)$ and $P_{2}(k)$ matrices are presented, respectively. It is observed that the convergence of $P_{1}(k)$ and 
Figure 3: Evolution of the complete parameters for the $P_{1}$ matrix.

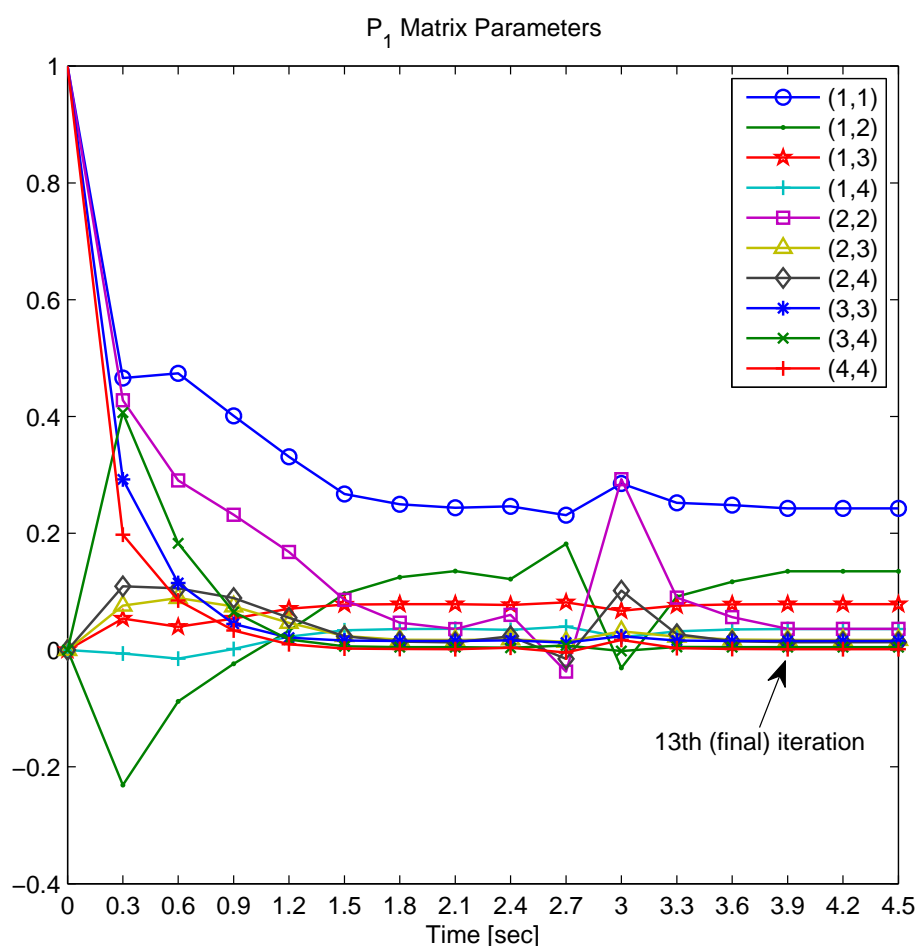

Figure 4: Evolution of the complete parameters for the $P_{2}(k)$ matrix.

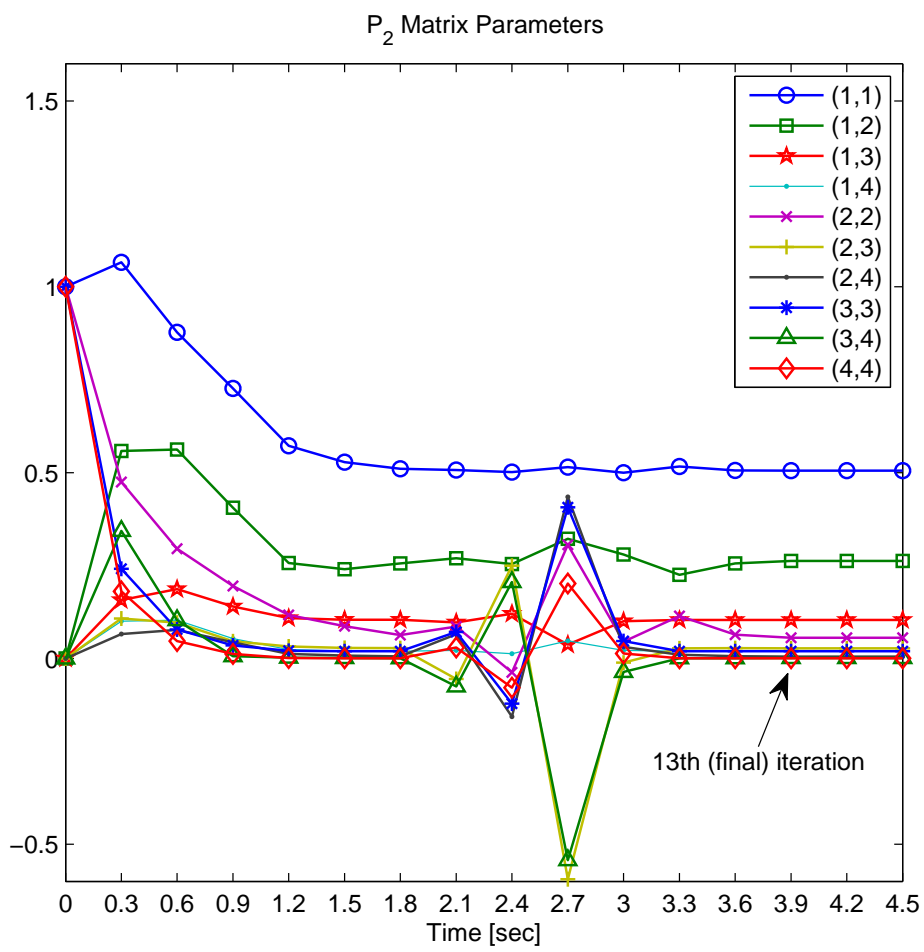


Figure 5: The state error of $x(t)-x^{*}(t)$ during the simulation.

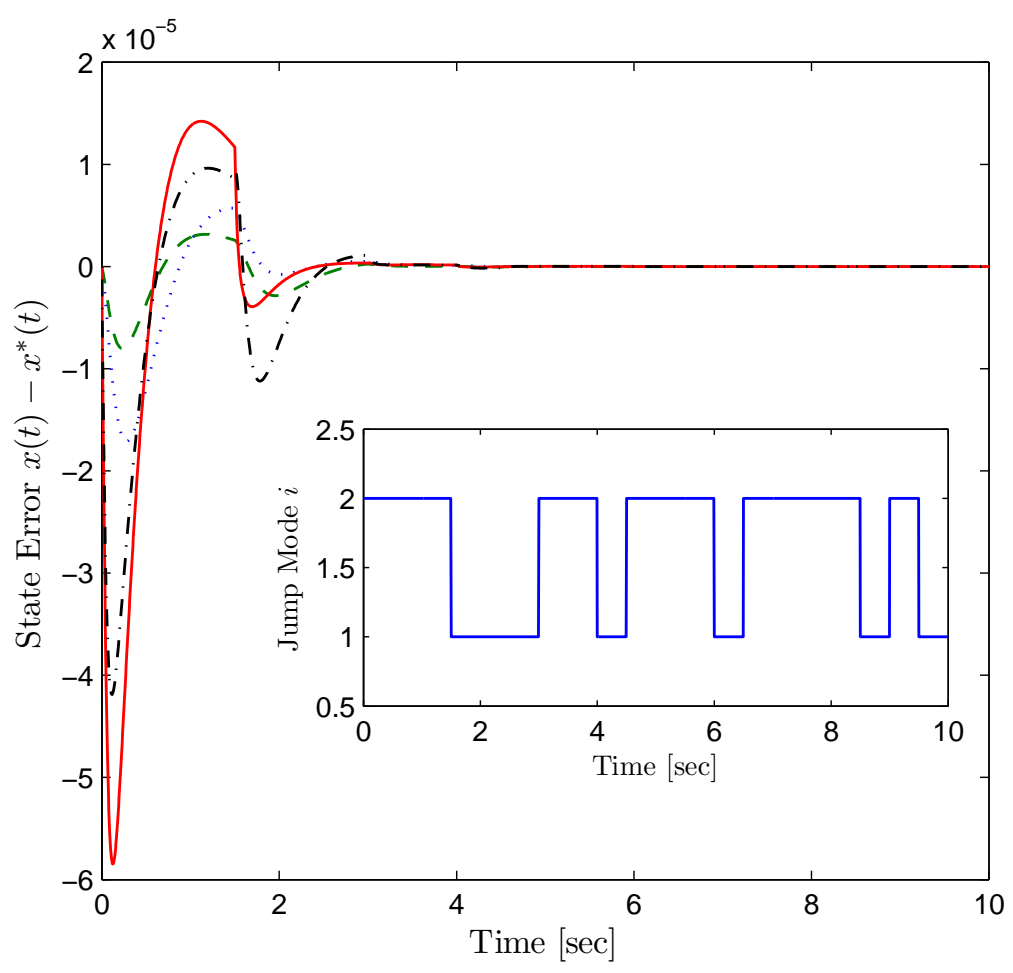

$P_{2}(k)$ to their optimal values in the $10^{-2}$ accuracy at time $3.9 \sec$ (i.e. 13th iteration). With the simulation time $t \in\left[\begin{array}{ll}0 & 10 \mathrm{sec}\end{array}\right]$ and the jumping time $\Delta t=0.5 \mathrm{sec}$, the responses of the state errors $x(t)-x^{*}(t)$ between the system controlled by our obtained controller and the system controlled by the actual optimal controller are depicted in Figure 5. One can see from Figure 5 that the state errors are in the range of $10^{-4}$. This verifies that, by using the novel policy iteration algorithm in this paper, the JLQ optimal control problem for continuous-time MJLSs can be solved online efficiently without using the exact information of the continuous-time MJLSs matrices $A_{i}$.

Remark 9. It is observed that there are some obvious irregular perturbations in the convergence processes of $P_{1}(k)$ (see $2.7 \mathrm{sec} \sim 3.3 \mathrm{sec}$ in Figure 3) and $P_{2}(k)$ (see 2.1sec $\sim 3 \mathrm{sec}$ in Figure 4) matrices. This is because the persistently exciting condition required for solving parallel least-square sense (35) becomes very weak (perhaps even lost) along with the system state converges to almost zero. An effective method to prevent the potential perturbations is to add some small excitation signal (or some additive exploration noise) in the control input $u_{i}(t)$. For more details to this technique, we refer the readers to [36][7][29], and the references therein.

\section{Conclusions}

In this paper, we proposed a novel online policy iteration algorithm to solve the JLQ optimal control problem for the continuous-time MJLSs without the exact knowledge of the system matrices. The algorithm was obtained by a new decoupling technique named Subsystems Transformation with a set of new continuous-time Markov jump linear subsystems. Actually, this method is equivalent to online solve the coupled algebraic matrix Riccati 
equations by utilizing the sampling information of the subsystems states. The convergence of the novel policy iteration algorithm is proved. Simulation results also illustrate the effectiveness and applicability of the proposed approach.

\section{References}

[1] Borno, I.: 'Parallel computation of the solutions of coupled algebraic Lyapunov equations', Automatica, 1995, 31, (9), pp. 1345-1347

[2] Gajic, Z., Borno, I.: 'Lyapunov iterations for optimal control of jump linear systems at steady state', IEEE Transactions on Automatic Control, 1995, 40, (11), pp. 1971-1975

[3] Salama, A., Gourishankar, V.: 'A computational algorithm for solving a system of coupled algebraic matrix Riccati equations', IEEE Transactions on Computers, 1974, 23, (1), pp. 100-102

[4] Ivanov, I.G.: 'On some iterations for optimal control of jump linear equations', Nonlinear Analysis: Theory, Method \& Applications, 2008, 69, (11), pp. 4012-4024

[5] Wu, H., Luo, B.: 'Simultaneous policy update algorithms for learning the solution of linear continuous-time $H_{\infty}$ state feedback control', Information Sciences, 2013, 222, pp. 472-485

[6] Vrabie, D., Pastrvanu, O., Abu-Khalaf, M., Lewis F.: 'Adaptive optimal control for continuous-time linear systems based on policy iteration', Automatica, 2009, 45, (2), pp. 477-484

[7] Jiang, Y., Jiang, Z.: 'Computational adaptive optimal control for continuous-time linear systems with completely unknown dynamics', Automatica, 2012, 48, (10): pp. 2699-2704

[8] Ji, Y., Chizeck, H.J.: 'Controllability, stabilizability, and continuous-time Markovian jump linear quadratic control', IEEE Transactions on Automatic Contol, 1990, 35, (7), pp. 777-788

[9] Sworder, D.D.: 'Feedback control of a class of linear systems with jump parameters', IEEE Transactions on Automatic Contol, 1969, 14, (1), pp. 9-14

[10] Borno, I., Gajic, Z.: 'Parallel algorithm for solving coupled algebraic Lyapunov equations of discrete-time jump linear systems', Computers \& Mathematics with Applications, 1995, 30, (7), pp. 1-4

[11] Zhou, B., Lam, J., Duan, G.: 'Convergence of gradient-based iterative solution of coupled Markovian jump Lyapunov equations', Computers \& Mathematics with Applications, 2008, 56, (12), pp. 3070-3078

[12] Krasovskii, N.N., Lidskii, E.A.: 'Analytical design of controllers in systems with random attributes', 1961, 22, (1-3), pp. 1021-1025

[13] Robinson, V., Sworder, D.: 'A computational algorithm for design of regulators for linear jump parameter systems', IEEE Transactions on Automatic Control, 1974, 19, (1), pp. 47-49

[14] Kleinman, D.: 'On an iterative technique for Riccati equation computations', IEEE Transactions on Automatic Control, 1968, 13, (1), pp. 114-115 
[15] Zhang, H., Wei, Q., Luo, Y.: 'A novel infinite-time optimal tracking control scheme for a class of discretetime nonlinear systems via the greedy HDP iteration algorithm', IEEE Transactions on Systems, Man, and Cybernetics, Part B: Cybernetics, 2008, 38, (4), pp. 937-942

[16] Bartels, R.H., Stewart, G.W.: 'Solution of the matrix equation $A X+B X=C$ ', Communications of ACM, 1972, 15, (9), pp. 820-826

[17] Golub, G.H., Nash, S., Van Loan C.F.: 'A Hessenberg-Schur method for the problem $A X+X B=C$ ', IEEE Transactions on Automatic Control, 1979, 24, (6), pp. 909-913

[18] Abu-Khalaf, M., Lewis, F.: 'Nearly optimal control laws for nonlinear systems with saturating actuators using a neural network HJB approach', Automatica, 41, (5), pp. 779-791

[19] Li, Z., Zhou, B., Lam, J., Wang, Y.: 'Positive operator based iterative algorithms for solving Lyapunov equations for Itô stochastic systems with Markovian jumps', Applied Mathematics and Computation, 2011, 217, (21), pp. 8179-8195

[20] Damm, T., Hinrichsen, D.: 'Newton's method for a rational matrix equation occurring in stochastic control', Linear Algebra and its Applications, 2001, 332, pp. 81-109

[21] Murray, J.J., Cox, C.J., Lendaris, G.G., Saeks, R.: 'Adaptive dynamic programming', IEEE Transactions on Systems, Man, and Cybernetics-Part C: Applications and Reviews, 2002, 32, (2), pp. 140-153

[22] Chen, B., Niu, Y., Zou, Y.: 'Adaptive sliding mode control for stochastic Markovian jumping systems with actuator degradation', Automatica, 2013a, 49, (6), pp. 1748-1754

[23] Zhang, L., Boukas, E.: 'Stability and stabilization of Markovian jump linear systems with partly unknown transition probabilities', Automatica, 2009, 45, (2), pp. 463-468

[24] Dragan, V., Morozan, T.: 'The linear quadratic optimization problems for a class of linear stochastic systems with multiplicative white noise and Markovian jumping', IEEE Transactions on Automatic Control, 2004, 49, (5), pp. 665-675

[25] Chen, B., Niu, Y., Zou, Y.: 'Sliding mode control for stochastic Markovian jumping systems with incomplete transition rate', IET Control Theory \& Applications, 2013, 7, (10), pp. 1330-1338

[26] Al-Tamimi, A., Lewis, F., Abu-Khalaf, M.: 'Discrete-time nonlinear HJB solution using approximate dynamic programming: convergence proof', IEEE Transactions on Systems, Man, and Cybernetics, Part B: Cybernetics, 2008, 38, (4), pp. 943-949

[27] Bhasin, S., Kamalapurkar, R., Johnson, M., Vamvoudakis, K.G., Lewis, F., Dixon, W.E.: 'A novel actorcritic-identifier architecture for approximate optimal control of uncertain nonlinear systems', Automatica, 2013, 49, (1), pp. 82-92

[28] Zhang, H., Lewis, F.: 'Adaptive cooperative tracking control of higher-order nonlinear systems with unknown dynamics', Automatica, 2012, 48, (7), pp. 1432-1439

[29] Vamvoudakis, K.G., Lewis, F.: 'Online solution of nonlinear two-player zero-sum games using synchronous policy iteration', International Journal of Robust and Nonlinear Control, 2012, 22, (13), pp. 1460-1483 
[30] Gajic, Z., Borno, I.: 'General transformation for block diagonalization of weakly coupled linear systems composed of $N$-subsystems', IEEE Tansactions on Circuits and Systems-I: Fundamental Theory and Applications, 2000, 47, (6), pp. 909-912

[31] Costa, O.L.V., Fragoso, M.D.: 'Discrete-time LQ-optimal control problems for infinite Markov jump parameter systems', IEEE Transactions on Automatic Control, 1995, 40, (12), pp. 2076-2088

[32] Costa, O.L.V., do Val, J.B.R., Geromel J.C.: 'Continuous-tme state-feedback $H_{2}$-control of Markovian jump linear systems via convex analysis', Automatica, 1999, 35, (2), pp. 259-268

[33] Modares, H., Lewis, F.: 'Online solution to the linear quadratic tracking problem of continuous-time systems using reinforement learning', IEEE 52nd Annual Conference on Decision and Control (CDC), 2013, pp. 38513856

[34] Wonham, M.: 'On a matrix Riccati equation of stochastic control', SIAM Journal on Control, 1968, 6, (4), pp. 681-697

[35] Gajic, Z., Losada, R.: 'Monotonicity of algebraic Lyapunov iterations for optimal control of jump parameter linear systems', Systems \& Control Letter, 2000, 41, (3), pp. 175-181

[36] Lee, J.Y., Park, J.B., Choi, Y.H.: 'Policy-iteration-based adaptive optimal control for uncertain continuoustime linear systems with excitation signals', International Conference on Control, Automation and Systems, 2010, pp. 646-651

[37] Lewis, F.L., Vrabie, D.: 'Reinforcement learning and adaptive dynamic programming for feedback control', IEEE Transactions on Circuits and Systems Magazine, 2009, 9, (3), pp. 32-50

[38] Sutton, R.S., Barto, A.G.: 'Reinforcement learning: an introduction', MIT Press, Cambridge MA, 1998.

[39] Vrabie, D., Lewis, F.: 'Online adaptive optimal control based on reinforcement learning', Optimization and Optimal Control, Springer New York, 2010, pp. 309-323

[40] Vrabie, D., Lewis, F.: 'Neural network approach to continuous-time direct adaptive optimal control for partially unknown nonlinear systems', Neural Networks, 2009, 22, (3), pp. 237-246

[41] Wang, F., Zhang, H., Liu, D.: 'Adaptive dynamic programming: an introduction', IEEE Transactions on Computational Intelligence Magazine, 2009, 4, (2), pp. 39-47

[42] He, P., Jagannathan, S.: 'Reinforcement learning neural-network-based controller for nonlinear discrete-time systems with input constraints'. IEEE Transactions on Systems, Man, and Cybernetics, Part B: Cybernetics, 2007, 37, (2), pp. 425-436

[43] Howard, R.A.: 'Dynamic programming and Markov processes', MIT Press, Cambridge, MA, 1960. 